\title{
Common fixed point of multifunctions theorems in Cone metric spaces
}

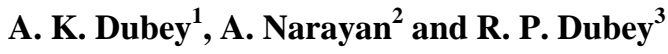 \\ ${ }^{1,2}$ Department of Mathematics, Bhilai Institute of Technology, Bhilai House, Durg 491001, India \\ ${ }^{3}$ Department of Mathematics, Dr.C.V.Raman University, Bilaspur, India \\ *Corresponding author E-mail: anilkumardby@ rediffmail.com
}

\begin{abstract}
In this paper, we generalize and obtain common fixed point of multifunctions in cone metric spaces. Our theorems improve and generalize of the results ([3],[9] and [10]).
\end{abstract}

Keywords: Complete cone metric space,multifunctions, common fixed point,normal constant.

\section{Introduction}

Recently, Guang and Xian [1] introduce the notion of cone metric spaces. He replaced real number system by ordered Banach space. He also gave the condition in the setting of cone metric spaces. These authors also described the convergence of sequences in the cone metric spaces and introduce the corresponding notion of completeness. In the setting of cone metric spaces, we improve and generalize multifunctions theorems and obtained common fixed point with normal constant $\mathrm{K}=1$.

\section{Preliminaries}

Let $E$ be a real Banach space and $P$ a subset of $E$. $P$ is called a cone if and only if

i) $P$ is closed, non-empty, and $P \neq\{0\}$,

ii) $a x+b y \in P$ for all $x, y \in P$ and non-negative real numbers $a, b$,

iii) $x \in P$ and $-x \in P \Rightarrow x=0$.

For a given cone $P \subseteq E$, we can define a partial ordering $\leq$ with respect to $P$ by $x \leq y$ if and only if $y-x \in P$. $x<$ $y$ will stand for $x \leq y$ and $x \neq y$, while $x \ll y$ will stand for $y-x \in$ int $P$, int $P$ denotes the interior of $P$ [1].

The cone $P$ is called normal if there is a number $K>0$ such that for all $x, y \in E$

$0 \leq x \leq y$ implies $\|x\| \leq K\|y\|$.

The least positive number $K$ satisfying the above is called the normal constant of $P$ [1]. It is clear that $K \geq 1$.

In the following, we always suppose that $E$ is a normed space, $P$ is a cone in $E$ with normal constant $K=1$, int $P \neq \emptyset$ and $\leq$ is partial ordering with respect to $P$.

Definition 2.1. Let $X$ be a non-empty set. Suppose that the map $d: X \times X \rightarrow E$ satisfies

(i) $\quad 0 \leq d(x, y)$ for all $x, y \in X$, and $d(x, y)=0$ if and only if $x=y$;

(ii) $\quad d(x, y)=d(y, x)$ for all $x, y \in X$;

(iii) $\quad d(x, y) \leq d(x, z)+d(z, y)$ for all $x, y, z \in X$.

Then $d$ is called a cone metric on $X$, and $(X, d)$ is called cone metric space [1].

Example 2.2 (see[1]). Let $E=\mathbb{R}^{2}, P=\{(x, y) \in E: x, y \geq 0\} \subset \mathbb{R}^{2}, X=\mathbb{R}$, and $d: X \times X \rightarrow E$ defined by $d(x, y)=(|x-y|, \propto|x-y|)$, where $\propto \geq 0$ is a constant. Then $(X, d)$ is a cone metric space, and the normal constant of $P$ is $K=1$. 
Example 2.3 (See[11]). Let $E=\ell^{1}, P=\left\{\left\{x_{n}\right\}_{\mathrm{n} \geq 1} \in E: x_{\mathrm{n}} \geq 0\right.$ for all $\left.n\right\},(X, \rho)$ a metric space and $d: X \times X \rightarrow E$ defined by $d(x, y)=\left(\rho(x, y) / 2^{n}\right)_{n \geq 1}$. Then $(X, d)$ is a cone metric space and the normal constant of $P$ is $K=1$.

Clearly, the above example show that class of cone metric spaces contains the class of metric spaces.

Definition 2.4 (see[1]). Let $(X, d)$ be a cone metric space, $x \in X$ and $\left\{x_{n}\right\}_{n \geq 1}$ a sequence in $X$. Then

(i) $\quad\left\{x_{n}\right\}_{\mathrm{n} \geq 1}$ converges to $x$ whenever for every $c \in E$ with $0 \ll c$ there is a natural number $N$ such that $d\left(x_{n}, x\right) \ll c$ for all $n \geq N$. We denote this by $\lim _{n \rightarrow \infty} x_{\mathrm{n}}=x$ or $x_{n} \rightarrow x$.

(ii) $\quad\left\{x_{n}\right\}_{\mathrm{n} \geq 1}$ is a Cauchy sequence whenever for every $c \in E$ with $0 \ll c$ there is a natural number $N$ such that $d\left(x_{n}, x_{m}\right) \ll c$ for all $n, m \geq N$.

(iii) $(X, d)$ is a complete cone metric space if every Cauchy sequence is convergent.

Most familiar cones are normal with normal constant $K=1$. But, for each $k>1$ there are cones with normal constant $K>k$. Also, there are non-normal cones [2].

Lemma 2.5 (See[3]). Let $(X, d)$ be a cone metric space, $P$ a normal cone with normal constant $K=1$, and $A$ a compact set in $\left(X, \tau_{c}\right)$. Then, for every $x \in X$ there exists $a_{0} \in A$ such that

$$
\left\|d\left(x, a_{o}\right)\right\|=\inf _{a \in A}\|d(x, a)\| .
$$

Lemma 2.6 (See[3]). Let $(X, d)$ be a cone metric space, $P$ a normal cone with normal constant $K=1$, and $A, B$ two compact sets in $\left(X, \tau_{c}\right)$. Then,

$$
\sup _{x \in B} d^{\prime}(x, A)<\infty,
$$

Where

$$
d^{\prime}(x, A)=\inf _{a \in A}\|d(x, a)\| .
$$

Definition 2.7 (See[3]). Let $(X, d)$ be a cone metric space, $P$ a normal cone with normal constant $K=1, H_{c}(X)$ the set of all compact subsets of $\left(X, \tau_{c}\right)$ and $A \in H_{c}(X)$. By using Lemma 2.6, we can define

By

$$
h_{A}: H_{c}(X) \rightarrow[0, \infty) \text { and } d_{H}: H_{c}(X) \times H_{c}(X) \rightarrow[0, \infty)
$$

respectively.

$$
h_{A}(B)=\sup _{x \in A} d^{\prime}(x, B) \text { and } d_{H}(A, B)=\max \left\{h_{A}(B), h_{B}(A)\right\}
$$

Remark 2.8 (See[3]). Let $(X, d)$ be a cone metric space with normal constant $K=1$, Define $\rho: X \times X \rightarrow[0, \infty)$ by $\rho(x, y)=\|d(x, y)\|$. Then, $(X, \rho)$ is a metric space. This implies that for each $A, B \in H_{c}(X)$ and $x, y \in X$, we have the following relations

(i) $\quad d^{\prime}(x, A) \leq\|d(x, y)\|+d^{\prime}(y, A)$,

(ii) $\quad d^{\prime}(x, A) \leq d^{\prime}(x, B)+h_{B}(A)$,

(iii) $\quad d^{\prime}(x, A) \leq\|d(x, y)\|+d^{\prime}(y, B)+h_{B}(A)$.

\section{Main results}

Theorem 3.1. Let $(X, d)$ be a complete cone metric space with normal constant $K=1$, and the multifunctions $T_{1}, T_{2}: X \rightarrow H_{c}(X)$ satisfy the relation

$$
d_{H}\left(T_{1} x, T_{2} y\right) \leq c\left(d^{\prime}\left(T_{1} x, x\right)+d^{\prime}\left(T_{2} y, y\right)\right)
$$

For all $x, y \in X$, where $c \in\left(0, \frac{1}{2}\right)$ is a constant.

Then, $T_{1}$ and $T_{2}$ have a common fixed point.

Proof. Let $x_{o} \in X$ be given $n \geq 1$. By Lemma 2.5, choose $x_{1} \in T_{1} x_{o}$ and $x_{2} \in T_{2} x_{1}$, such that $d^{\prime}\left(x_{o}, T_{1} x_{o}\right)=\left\|d\left(x_{o}, x_{1}\right)\right\|$ 
Similarly $d^{\prime}\left(x_{1}, T_{2} x_{1}\right)=\left\|d\left(x_{1}, x_{2}\right)\right\|$.

If $x_{2 n+1}$ and $x_{2 n+2}$ have been given, then choose $x_{2 n+2} \in T_{1} x_{2 n+1}$ and $x_{2 n+3} \in T_{2} x_{2 n+2}$ such that

$d^{\prime}\left(x_{2 n+1}, T_{1} x_{2 n+1}\right)=\left\|d\left(x_{2 n+1}, x_{2 n+2}\right)\right\|$

Similarly

$d^{\prime}\left(x_{2 n+2}, T_{2} x_{2 n+2}\right)=\left\|d\left(x_{2 n+2}, x_{2 n+3}\right)\right\|$

Now from (3.1),

For all $\mathrm{n} \geq 1$. Hence;

$$
\begin{aligned}
& \left\|d\left(x_{2 n+1}, x_{2 n+2}\right)\right\|=d^{\prime}\left(x_{2 n+1}, T_{1} x_{2 n+1}\right) \\
& \leq h_{T_{1} x_{2 n}}\left(T_{1} x_{2 n+1}\right) \\
& \leq d_{H}\left(T_{1} x_{2 n}, T_{1} x_{2 n+1}\right) \\
& \leq c\left(d^{\prime}\left(T_{1} x_{2 n}, x_{2 n}\right)+d^{\prime}\left(T_{1} x_{2 n+1}, x_{2 n+1}\right)\right) \\
& =c\left(\left\|d\left(x_{2 n+1}, x_{2 n}\right)\right\|+\left\|d\left(x_{2 n+1}, x_{2 n+2}\right)\right\|\right) \text {, }
\end{aligned}
$$

$\left\|d\left(x_{2 n+1}, x_{2 n+2}\right)\right\| \leq \frac{c}{1-c}\left\|d\left(x_{2 n+1}, x_{2 n}\right)\right\|$,

For all $n \geq 1$. Put $\lambda=\frac{c}{1-c}$ in (3.3). Then, for $n>m$, we have

$$
\begin{aligned}
\left\|d\left(x_{2 n+1}, x_{2 m+1}\right)\right\| & \leq \sum_{i=2 m+2}^{2 n+1}\left\|d\left(x_{i}, x_{i-1}\right)\right\| \\
& \leq\left(\lambda^{2 n}+-----\lambda^{2 m+1}\right)\left\|d\left(x_{o}, x_{1}\right)\right\| \\
& \leq \frac{\lambda^{2 m+1}}{1-\lambda}\left\|d\left(x_{o}, x_{1}\right)\right\| .
\end{aligned}
$$

This implies that

$$
\lim _{m, n \rightarrow \infty}\left\|d\left(x_{2 n+1}, x_{2 m+1}\right)\right\|=0 .
$$

By [1,Lemma 4], $\left\{x_{2 n+1}\right\}_{n \geq 1}$ is a Cauchy sequence in $X$. Thus there exists $x^{*} \in X$ such that $x_{2 n+1} \rightarrow x^{*}$. Now by using Remark 2.8, we have

For all $\mathrm{n} \geq 1$. Hence,

$$
\begin{aligned}
d^{\prime}\left(x^{*}, T_{1} x^{*}\right) & \leq d^{\prime}\left(x^{*}, T_{1} x_{2 n+1}\right)+h_{T_{1} x_{2 n+1}}\left(T_{1} x^{*}\right) \\
& \leq d^{\prime}\left(x^{*}, T_{1} x_{2 n+1}\right)+d_{H}\left(T_{1} x_{2 n+1}, T_{1} x^{*}\right) \\
& \leq\left\|d\left(x^{*}, x_{2 n+2}\right)\right\|+c\left(d^{\prime}\left(T_{1} x_{2 n+1}, x_{2 n+1}\right)+d^{\prime}\left(T_{1} x^{*}, x^{*}\right)\right)
\end{aligned}
$$

$\begin{aligned} d^{\prime}\left(x^{*}, T_{1} x^{*}\right) & \leq \frac{c}{1-c} d^{\prime}\left(T_{1} x_{2 n+1}, x_{2 n+1}\right)+\frac{1}{1-c}\left\|d\left(x^{*}, x_{2 n+2}\right)\right\| \\ & =\frac{c}{1-c}\left\|d\left(x_{2 n+2}, x_{2 n+1}\right)\right\|+\frac{1}{1-c}\left\|d\left(x^{*}, x_{2 n+2}\right)\right\|,\end{aligned}$

For all $n \geq 1$. Therefore $d^{\prime}\left(x^{*}, T_{1} x^{*}\right)=0$. By Lemma $2.5, x^{*} \in T_{1} x^{*}$.

Similarly, it can be established that $x^{*} \in T_{2} x^{*}$, that is, $x^{*}$ is a common fixed point of pair of $\mathrm{T}_{1}$ and $\mathrm{T}_{2}$.

Theorem 3.2. Let $(X, d)$ be complete cone metric space with normal constant $K=1$ and the multifunctions $T_{1}, T_{2}: X \rightarrow$ $H_{c}(X)$ satisfy the relation

$$
d_{H}\left(T_{1} x, T_{2} y\right) \leq c\left(d^{\prime}\left(T_{1} x, y\right)+d^{\prime}\left(T_{2} y, x\right)\right)
$$

For all $x, y \in X$, where $c \in\left(0, \frac{1}{2}\right)$ is a constant. Then $T_{1}$ and $T_{2}$ have a common fixed point.

Proof. Let $x_{o} \in X$ be given and $\mathrm{n} \geq 1$. By Lemma 2.5, choose $x_{1} \in T_{1} x_{o}$ and $x_{2} \in T_{2} x_{1}$ such that

Similarly

$$
d^{\prime}\left(x_{o}, T_{1} x_{o}\right)=\left\|d\left(x_{o}, x_{1}\right)\right\|
$$

$$
d^{\prime}\left(x_{1}, T_{2} x_{1}\right)=\left\|d\left(x_{1}, x_{2}\right)\right\| .
$$

If $x_{2 n+1}$ and $x_{2 n+2}$ have been given, then choose $x_{2 n+2} \in T_{1} x_{2 n+1}$ and $x_{2 n+3} \in T_{2} x_{2 n+2}$ such that

$$
d^{\prime}\left(x_{2 n+1}, T_{1} x_{2 n+1}\right)=\left\|d\left(x_{2 n+1}, x_{2 n+2}\right)\right\|
$$


Similarly

$d^{\prime}\left(x_{2 n+2}, T_{2} x_{2 n+2}\right)=\left\|d\left(x_{2 n+2}, x_{2 n+3}\right)\right\|$

Now from (3.4),

For all $n \geq 1$. Hence;

$$
\begin{aligned}
\left\|d\left(x_{2 n+1}, x_{2 n+2}\right)\right\| & =d^{\prime}\left(x_{2 n+1}, T_{1} x_{2 n+1}\right) \\
& \leq h_{T_{1} x_{2 n}}\left(T_{1} x_{2 n+1}\right) \\
& \leq d_{H}\left(T_{1} x_{2 n}, T_{1} x_{2 n+1}\right) \\
& \leq c\left(d^{\prime}\left(T_{1} x_{2 n}, x_{2 n+1}\right)+d^{\prime}\left(T_{1} x_{2 n+1}, x_{2 n}\right)\right) \\
& \leq c\left(\left\|d\left(x_{2 n+1}, x_{2 n}\right)\right\|+\left\|d\left(x_{2 n+1}, x_{2 n+2}\right)\right\|\right),
\end{aligned}
$$

$\left\|d\left(x_{2 n+1}, x_{2 n+2}\right)\right\| \leq \frac{c}{1-c}\left\|d\left(x_{2 n+1}, x_{2 n}\right)\right\|$,

For all $\mathrm{n} \geq 1$. Put $\lambda=\frac{c}{1-c}$ in (3.6). Then, for $\mathrm{n}>\mathrm{m}$, we have

$\left\|d\left(x_{2 n+1}, x_{2 m+1}\right)\right\| \leq \sum_{i=2 m+2}^{2 n+1}\left\|d\left(x_{i}, x_{i-1}\right)\right\|$

$$
\begin{aligned}
& \leq\left(\lambda^{2 n}+-----+\lambda^{2 m+1}\right)\left\|d\left(x_{o}, x_{1}\right)\right\| \\
& \leq \frac{\lambda^{2 m+1}}{1-\lambda}\left\|d\left(x_{o}, x_{1}\right)\right\| .
\end{aligned}
$$

This implies that

$$
\lim _{m, n \rightarrow \infty}\left\|d\left(x_{2 n+1}, x_{2 m+1}\right)\right\|=0 .
$$

By [1,Lemma 4], $\left\{x_{2 n+1}\right\}_{\mathrm{n} \geq 1}$ is a Cauchy sequence in $X$. Thus there exists $x^{*} \in X$ such that $x_{2 n+1} \rightarrow x^{*}$. Now by using Remark 2.8, we have

$$
\begin{aligned}
d^{\prime}\left(x^{*}, T_{1} x^{*}\right) & \leq d^{\prime}\left(x^{*}, T_{1} x_{2 n+1}\right)+h_{T_{1} x_{2 n+1}}\left(T_{1} x^{*}\right) \\
& \leq d^{\prime}\left(x^{*}, T_{1} x_{2 n+1}\right)+d_{H}\left(T_{1} x_{2 n+1}, T_{1} x^{*}\right) \\
& \leq\left\|d\left(x^{*}, x_{2 n+2}\right)\right\|+c\left(d^{\prime}\left(T_{1} x_{2 n+1}, x^{*}\right)+d^{\prime}\left(T_{1} x^{*}, x_{2 n+1}\right)\right) \\
& \leq\left\|d\left(x^{*}, x_{2 n+2}\right)\right\|+c\left(\left\|d\left(x^{*}, x_{2 n+2}\right)\right\|+\left\|d\left(x_{2 n+1}, x^{*}\right)\right\|+d^{\prime}\left(T_{1} x^{*}, x^{*}\right)\right)
\end{aligned}
$$

For all $n \geq 1$. Hence

$$
d^{\prime}\left(x^{*}, T_{1} x^{*}\right) \leq \frac{1+c}{1-c}\left\|d\left(x^{*}, x_{2 n+2}\right)\right\|+\frac{1}{1-c}\left\|d\left(x_{2 n+1}, x^{*}\right)\right\|
$$

For all $\mathrm{n} \geq 1$. Therefore, $d^{\prime}\left(x^{*}, T_{1} x^{*}\right)=0$. By Lemma $2.5, x^{*} \in T_{1} x^{*}$.

Similarly, it can be established that $x^{*} \in T_{2} x^{*}$, that is $x^{*}$ is a common fixed point of pair of $T_{1}$ and $T_{2}$.

Theorem 3.3 Let $(X, d)$ be a complete cone metric space with normal constant $K=1$ and the multifunctions $T_{1}, T_{2}: X \rightarrow H_{c}(X)$ satisfy the relation

$$
\begin{aligned}
d_{H}\left(T_{1} x, T_{2} y\right) & \leq a\left[d^{\prime}\left(x, T_{1} x\right)+d^{\prime}\left(y, T_{2} y\right)\right] \\
& +b\left[d^{\prime}\left(x, T_{2} y\right)+d^{\prime}\left(y, T_{1} x\right)\right] \\
& +c\left[d^{\prime}(x, y)+d^{\prime}\left(T_{1} x, T_{2} y\right)\right]
\end{aligned}
$$

For all $x, y \in X$ and $\mathrm{a}+\mathrm{b}+\mathrm{c}<\frac{1}{2} \mathrm{a}, \mathrm{b}, \mathrm{c} \in\left[0, \frac{1}{2}\right)$ are constants. Then $T_{1}$ and $T_{2}$ have a common fixed point.

Proof. Let $x_{o} \in X$ be given and $\mathrm{n} \geq 1$. By Lemma 2.5, choose $x_{1} \in T_{1} x_{o}$ and $x_{2} \in T_{2} x_{1}$ such that

$$
d^{\prime}\left(x_{o}, T_{1} x_{o}\right)=\left\|d\left(x_{o}, x_{1}\right)\right\|
$$

Similarly

$$
d^{\prime}\left(x_{1}, T_{2} x_{1}\right)=\left\|d\left(x_{1}, x_{2}\right)\right\|
$$

If $x_{2 n+1}$ and $x_{2 n+2}$ have been given, then choose $x_{2 n+2} \in T_{1} x_{2 n+1}$ and $x_{2 n+3} \in T_{2} x_{2 n+2}$ such that

$d^{\prime}\left(x_{2 n+1}, T_{1} x_{2 n+1}\right)=\left\|d\left(x_{2 n+1}, x_{2 n+2}\right)\right\|$

Similarly

$d^{\prime}\left(x_{2 n+2}, T_{2} x_{2 n+2}\right)=\left\|d\left(x_{2 n+2}, x_{2 n+3}\right)\right\|$ 
Now from (3.7),

$$
\begin{aligned}
& \left\|d\left(x_{2 n+1}, x_{2 n+2}\right)\right\|=d^{\prime}\left(x_{2 n+1}, T_{1} x_{2 n+1}\right) \\
& \leq h_{T_{1} x_{2 n}}\left(T_{1} x_{2 n+1}\right) \\
& \leq d_{H}\left(T_{1} x_{2 n}, T_{1} x_{2 n+1}\right) \\
& \leq a\left[d^{\prime}\left(x_{2 n}, T_{1} x_{2 n}\right)+d^{\prime}\left(x_{2 n+1}, T_{1} x_{2 n+1}\right)\right] \\
& +b\left[d^{\prime}\left(x_{2 n}, T_{1} x_{2 n+1}\right)+d^{\prime}\left(x_{2 n+1}, T_{1} x_{2 n}\right)\right] \\
& +c\left[d^{\prime}\left(x_{2 n}, x_{2 n+1}\right)+d^{\prime}\left(T_{1} x_{2 n}, T_{1} x_{2 n+1}\right)\right] \\
& \leq a\left[d^{\prime}\left(x_{2 n}, x_{2 n+1}\right)+d^{\prime}\left(x_{2 n+1}, x_{2 n+2}\right)\right] \\
& +b\left[d^{\prime}\left(x_{2 n}, x_{2 n+2}\right)+d^{\prime}\left(x_{2 n+1}, x_{2 n+1}\right)\right] \\
& +c\left[d^{\prime}\left(x_{2 n}, x_{2 n+1}\right)+d^{\prime}\left(x_{2 n+1}, x_{2 n+2}\right)\right] \\
& \leq a\left[d^{\prime}\left(x_{2 n}, x_{2 n+2}\right)\right]+b\left[d^{\prime}\left(x_{2 n}, x_{2 n+2}\right)\right] \\
& +c\left[d^{\prime}\left(x_{2 n}, x_{2 n+2}\right)\right] \\
& \leq(a+b+c)\left[d^{\prime}\left(x_{2 n}, x_{2 n+2}\right)\right] \\
& \leq(a+b+c)\left[d^{\prime}\left(T_{1} x_{2 n+1}, x_{2 n}\right)\right] \\
& \leq(a+b+c)\left(\left\|d\left(x_{2 n+2}, x_{2 n}\right)\right\|\right) \\
& \leq(a+b+c)\left(\left\|d\left(x_{2 n}, x_{2 n+1}\right)\right\|+\left\|d\left(x_{2 n+1}, x_{2 n+2}\right)\right\|\right)
\end{aligned}
$$

For all $n \geq 1$. Hence

$\left\|d\left(x_{2 n+1}, x_{2 n+2}\right)\right\| \leq \frac{a+b+c}{1-(a+b+c)}\left\|d\left(x_{2 n+1}, x_{2 n}\right)\right\|$

For all $\mathrm{n} \geq 1$. Put $\lambda=\frac{a+b+c}{1-(a+b+c)}$ in (3.9). Then, for $\mathrm{n}>\mathrm{m}$, we have

$$
\begin{aligned}
\left\|d\left(x_{2 n+1}, x_{2 m+1}\right)\right\| & \leq \sum_{i=2 m+2}^{2 n+1}\left\|d\left(x_{i}, x_{i-1}\right)\right\| \\
& \leq\left(\lambda^{2 n}+-----+\lambda^{2 m+1}\right)\left\|d\left(x_{o}, x_{1}\right)\right\| \\
& \leq \frac{\lambda^{2 m+1}}{1-\lambda}\left\|d\left(x_{o}, x_{1}\right)\right\| .
\end{aligned}
$$

This implies that

$$
\lim _{m, n \rightarrow \infty}\left\|d\left(x_{2 n+1}, x_{2 m+1}\right)\right\|=0 .
$$

By [1,Lemma 4], $\left\{x_{2 n+1}\right\}_{\mathrm{n} \geq 1}$ is a Cauchy sequence in $X$. Thus there exists $x^{*} \in X$ such that $x_{2 n+1} \rightarrow x^{*}$. Now by using Remark 2.8, we have

$$
\begin{aligned}
d^{\prime}\left(x^{*}, T_{1} x^{*}\right) & \leq d^{\prime}\left(x^{*}, T_{1} x_{2 n+1}\right)+h_{T_{1} x_{2 n+1}}\left(T_{1} x^{*}\right) \\
& \leq d^{\prime}\left(x^{*}, T_{1} x_{2 n+1}\right)+d_{H}\left(T_{1} x_{2 n+1}, T_{1} x^{*}\right) \\
& \leq\left\|d\left(x^{*}, x_{2 n+2}\right)\right\|+a\left[d^{\prime}\left(x_{2 n+1}, T_{1} x_{2 n+1}\right)+d^{\prime}\left(x^{*}, T_{1} x^{*}\right)\right] \\
& +b\left[d^{\prime}\left(x_{2 n+1}, T_{1} x^{*}\right)+d^{\prime}\left(x^{*}, T_{1} x_{2 n+1}\right)\right] \\
& +c\left[d^{\prime}\left(x_{2 n+1}, x^{*}\right)+d^{\prime}\left(T_{1} x_{2 n+1}, T_{1} x^{*}\right)\right] \\
& \leq\left\|d\left(x^{*}, x_{2 n+2}\right)\right\|+a\left[d^{\prime}\left(x_{2 n+1}, x_{2 n+2}\right)+d^{\prime}\left(x^{*}, T_{1} x^{*}\right)\right] \\
& +b\left[d^{\prime}\left(x_{2 n+1}, x^{*}\right)+d^{\prime}\left(x^{*}, T_{1} x^{*}\right)+d^{\prime}\left(x^{*}, x_{2 n+2}\right)\right] \\
& +c\left[d^{\prime}\left(x_{2 n+1}, x^{*}\right)+d^{\prime}\left(x_{2 n+2}, T_{1} x^{*}\right)\right] \\
& \leq\left\|d\left(x^{*}, x_{2 n+2}\right)\right\|+a\left[d^{\prime}\left(x_{2 n+1}, x_{2 n+2}\right)+d^{\prime}\left(x^{*}, T_{1} x^{*}\right)\right] \\
& +b\left[d^{\prime}\left(x_{2 n+1}, x^{*}\right)+d^{\prime}\left(x^{*}, T_{1} x^{*}\right)+d^{\prime}\left(x^{*}, x_{2 n+2}\right)\right] \\
& +c\left[d^{\prime}\left(x_{2 n+1}, T_{1} x^{*}\right)+d^{\prime}\left(x^{*}, T_{1} x^{*}\right)+d^{\prime}\left(T_{1} x^{*}, x_{2 n+2}\right)\right] \\
(1-(a+b+c)) d & d^{\prime}\left(x^{*}, T_{1} x^{*}\right) \leq(a+b+c)\left[d^{\prime}\left(x_{2 n+1}, x_{2 n+2}\right)\right] \\
& \quad+\left\|d\left(x^{*}, x_{2 n+2}\right)\right\| \\
& +\frac{1}{1-(a+b+c)}\left\|d\left(x^{*}, x_{2 n+2}\right)\right\|
\end{aligned}
$$

For all $\mathrm{n} \geq 1$. Therefore, $d^{\prime}\left(x^{*}, T_{1} x^{*}\right)=0$. By Lemma $2.5, x^{*} \in T_{1} x^{*}$.

Similarly, it can be established that $x^{*} \in T_{2} x^{*}$, that is $x^{*}$ is a common fixed point of pair of $T_{1}$ and $T_{2}$. 
Corollary 3.4. Let $(X, d)$ be a complete cone metric space with normal constant $K=1$ and the multifunctions $T_{1}, T_{2}: X \rightarrow H_{c}(X)$ satisfy the relation

$$
\begin{aligned}
d_{H}\left(T_{1} x, T_{2} y\right) & \leq a\left[d^{\prime}\left(x, T_{1} x\right)+d^{\prime}\left(y, T_{2} y\right)\right] \\
& +b\left[d^{\prime}\left(x, T_{2} y\right)+d^{\prime}\left(y, T_{1} x\right)\right]
\end{aligned}
$$

For all $x, y \in X$ and $\mathrm{a}, \mathrm{b} \in\left[0, \frac{1}{2}\right)$ are constants. Then $T_{1}$ and $T_{2}$ have a common fixed point.

Proof: The Proof of the corollary immediately follows by putting $\mathrm{c}=0$ in the previous theorem 3.3 .

\section{References}

[1] Huang Gaung and Zhang Xian, “Cone metric spaces and fixed point theorems of contractive mappings” J. Math Anal. Appl.332(2007),14681476.

[2] Sh. Rezapour and R. Hamlbarani, "Some notes on the Paper-Cone metric Spaces and fixed point theorems of contractive mappings", J. Math. Anal. Appl.345 (2008),719-724.

[3] Sh. Rezapour and R.H.Haghi, "Fixed point of Multifunctions on Cone Metric Spaces", Numerical Functional Analysis and Optimization, 30:7-8(2009),825-832.

[4] H.Mohebi, "Topical functions and their properties in a class of ordered Banach spaces, in continuous optimization", Current Trends and Modern Applications, Part II, Springer (2005), PP 343-361.

[5] H.E.Kunze, D.La Torre and E.R. Vrscay, "Contraction multifunctions, fixed point inclusions and iterated multifunction system", J. Math. Anal. Appl. 330(2007):159-173.

[6] H.Covitz and S.B.Nadler," Multi-valued contraction mappings in generalized metric spaces”, Israel J. Math. 8(1970):5-11.

[7] F.S. De Blasi and P.Gr.Georgiev,"On a fixed point theorem of Ky Fan", Acta Math. Sinica 18(2)(2002):363-374.

[8] Seong-Hoon Cho and Mi Sun Kim, "Fixed Point theorems for general contractive multivalued mappings", J.Appl. Math. Informatics Vol.27 (2009), 343-350.

[9] R. Krishnakumar and M. Marudai, "Fixed Point Theorems of Multivalued Mappings in Cone Metric Spaces", Int.J. Contemp. Math. Sciences Vol.5, 2010, no.31,1533-1540.

[10] S. Jain and V.H.Badshah, "Fixed Point Theorem of Multivalued Mappings in Cone Metric Spaces", Int.J.Mathematical Archive2(12),2011,2753-2756.

[11] Sh.Rezapour, M.Derafshpour and R. Hamlbarani, "A review on topological properties of cone metric spaces", in Proceedings of the International Conference on Analysis, Topology and Applications (ATA'08), Vrinjacka Banja, Serbia, May-June 2008. 